\title{
BLOW UP NEAR HIGHER MODES OF NONLINEAR WAVE EQUATIONS
}

\author{
NATALIA STERNBERG
}

\begin{abstract}
This paper is concerned with the instability properties of higher modes of the nonlinear wave equation $u_{t t}-\Delta u-f(u)=0$ defined on a smoothly bounded domain with Dirichlet boundary conditions. It is shown that they are unstable in the sense that in any neighborhood of a higher mode there exists a solution of the given equation which blows up in finite time.
\end{abstract}

Introduction. The present paper is concerned with the instability properties of steady states of the nonlinear wave equation $u_{t t}-\Delta u-f(u)=0$, defined on a smoothly bounded domain $D \subset \mathbf{R}^{n}$ with Dirichlet boundary conditions. The steady states are solutions of the elliptic equations $-\Delta u-f(u)=0$ on $D$ with Dirichlet boundary conditions.

A number of authors have studied conditions on the initial data and the nonlinearity $f$ for which the solutions of the given wave equation blow up in finite time (see [13] for references). A blow up result for the positive solution of the elliptic equation (the ground state) was given by L. E. Payne and D. H. Sattinger in [13]. However, the instability of higher modes of nonlinear wave equations remained an open question. In this paper we show that for a general class of nonlinearities $f$ in any neighborhood of a steady state we can find such initial data for which the solution of the given wave equation blows up in finite time.

In [13] L. E. Payne and D. H. Sattinger were able to find an invariant region such that any solution of the nonlinear wave equation with initial data in this region blows up in finite time. Any attempt to find such an invariant region for a higher mode seems to fail. However, we can approximate higher modes by initial data of solutions which blow up in finite time.

The existence of infinitely many solutions of elliptic boundary value problems on a bounded domain was studied in a number of papers (see, for example, [2, 3, $\mathbf{5}, \mathbf{7}, \mathbf{8}, \mathbf{1 4}]$ ). We use the approach, given by A. Ambrosetti and P. H. Rabinowitz in [3], which is based on an application of the Ljusternik-Schnirelman category theory. The main idea is to consider the solutions of the elliptic equation as critical points of a functional. In our case, they are critical points of the restriction of a functional to an appropriate infinite-dimensional $C^{2}$-hypersurface, and therefore we can use the Ljusternik-Schnirelman category theory on Finsler manifolds (see [11]). The critical values are obtained using a minimum-maximum principle over sets of various genuses. The corresponding critical points are the steady states.

Received by the editors August 2, 1985.

1980 Mathematics Subject Classification (1985 Revision). Primary 35L05; Secondary 35B35. 
We prove the following:

THEOREM. Let $b_{k}$ be the critical value which is obtained using the minimummaximum principle over sets of genus $k \geq 2$. Then there exists an unstable steady state which corresponds to $b_{k}$. It is unstable in the sense that in any neighborhood of this steady state exists a solution of the given wave equation which blows up in finite time.

In the following we employ the following notation: \|\| is the norm in $H_{0}^{1}(D)$, defined by

$$
\|u\|^{2}=\int_{D}|\nabla u|^{2} d x
$$

\|\|$_{q}$ is the usual norm in $L^{q}(D)$; by $\rightarrow$ we denote weak convergence; and by $\rightarrow$ strong convergence.

The author would like to thank Professors Jack Hale and Walter Strauss for their helpful suggestions.

1. Preliminaries. Consider the nonlinear wave equation

$$
u_{t t}-\Delta u-f(u)=0
$$

on a smoothly bounded domain $D \subset \mathbf{R}^{n}$ with the Dirichlet boundary condition $u_{\mid \partial D}=0$.

The steady states of this equation are the nontrivial solutions of the elliptic equation

$$
-\Delta u-f(u)=0
$$

on $D$ with the boundary condition $u_{\mid \partial D}=0$.

Throughout this paper we will make the following assumptions:

(i) $F(z)=\int_{0}^{z} f(s) d s$

(ii) $f \in C^{2}(\mathbf{R}, \mathbf{R}), f(0)=f^{\prime}(0)=0$;

(iii) $f$ is strictly convex for $u>0$;

(iv) $f$ is odd;

(v) $u f^{\prime \prime}(u)=O\left(|u|^{q-2}\right)$;

(vi) $(p+1)|F(z)| \leq z f(z) \leq \varepsilon|z|^{2}+B|z|^{q}$, for all $z \in \mathbf{R}$, where $2<p+1 \leq q \leq$ $2 n /(n-2), \varepsilon, B>0$.

For example $f(u)=|u|^{2} u$.

Note that by (ii) and (iii) $\varepsilon$ is arbitrarily small.

The above assumptions imply that $z f(z)>0$, and $z\left(z f^{\prime}(z)-f(z)\right)>0$ for all $z \in \mathbf{R}-\{0\}$.

Consider the functionals $J, R: H_{0}^{1}(D) \rightarrow \mathbf{R}$, defined by

and

$$
J(u)=\frac{1}{2} \int_{D}|\nabla u|^{2} d x-\int_{D} F(u) d x
$$

Let

$$
R(u)=\int_{D}|\nabla u|^{2} d x-\int_{D} u f(u) d x
$$

$$
\tilde{M}=\left\{u \in H_{0}^{1}(D) \mid u \neq 0, R(u) \leq 0\right\} \quad \text { and } \quad M=\{u \in \tilde{M} \mid R(u)=0\} .
$$

The following proposition states some of the properties of $M, J$, and $R$ that will be useful in the future. 
PROPOSITION 1.1. (i) $\tilde{M}$ is bounded away from zero.

(ii) There exists a $K>0$ such that

$$
K\|u\| \leq J(u)-\frac{1}{2} R(u)<\infty
$$

for all $u \in \tilde{M}$. In particular,

$$
K\|u\| \leq J(u)<\infty
$$

for all $u \in M$.

(iii) The set $M$ is a $C^{2}$-submanifold of $H_{0}^{1}(D)$ of codimension 1 which contains all the nontrivial equilibrium solutions of $(1)$ in $H_{0}^{1}(D)$.

(iv) For any $u \in M$, the function $j: \mathbf{R}_{+} \rightarrow \mathbf{R}$, defined by $j(\alpha)=J(\alpha u)$, achieves its maximum at $\alpha=1$.

(v) For any $u \in \tilde{M}$ there exists an $\varepsilon>0$ such that the function $r$ from $(-\varepsilon+$ $1,1+\varepsilon)$ into $\mathbf{R}$, given by $r(\alpha)=R(\alpha u)$, is strictly monotone decreasing.

(vi) There exists an even continuous function $p$ from $H_{0}^{1}(D)-\{0\}$ into $(0, \infty)$ such that $R(p(u) u)=0$ for all $u \in H_{0}^{1}(D)-\{0\}$. If $u \in \tilde{M}$, then $0<p(u) \leq 1$.

(vii) For any $u \in \tilde{M}$ the function $g: \mathbf{R}_{+} \rightarrow \mathbf{R}$, defined by $g(\alpha)=J(\alpha)-\frac{1}{2} R(\alpha u)$, is strictly monotone increasing.

(viii) There exists a constant $S$ such that

$$
S<\int_{D} u\left(u f^{\prime}(u)-f(u)\right) d x<\infty
$$

for all $u \in \tilde{M}$.

ProOF. (i): Let $u \in \tilde{M}$ be arbitrary. Then $u \neq 0$, and

$$
0 \geq R(u)=\int_{D}|\nabla u|^{2} d x-\int_{D} u f(u) d x \geq\|u\|^{2}-\varepsilon\|u\|_{2}^{2}-B\|u\|_{q}^{q} .
$$

Using Sobolev's imbedding theorem we obtain that

$$
B K_{1}\|u\|^{2}\left(\left(1-\varepsilon K_{2}\right) / B K_{1}-\|u\|^{q-2}\right) \leq 0
$$

for some $K_{1}, K_{2}>0$. Therefore

$$
\|u\|^{q-2} \geq\left(1-\varepsilon K_{2}\right) / B K_{1}>0
$$

(ii): Let $u \in \tilde{M}$ be arbitrary. Then $R(u) \leq 0$. From part (i) and assumption (vi) we obtain that

$$
\begin{aligned}
J(u)-\frac{1}{2} R(u) & =\int_{D}\left(\frac{1}{2} u f(u)-F(u)\right) d x \\
& \geq \frac{p-1}{2(p+1)} \int_{D} u f(u) d x \geq \frac{p-1}{2(p+1)}\|u\|^{2}>0
\end{aligned}
$$

Furthermore,

$$
J(u)-\frac{1}{2} R(u)=\int_{D}\left(\frac{1}{2} u f(u)-F(u)\right) d x \leq \frac{p+3}{2(p+1)} \int_{D} u f(u) d x .
$$

Since the imbedding $H_{0}^{1}(D) \hookrightarrow L^{q}(D)$ is compact, it follows that $J(u)-\frac{1}{2} R(u)<\infty$.

(iii): Let $u$ be a nontrivial critical point of (1). Then, $-\Delta u-f(u)=0$. 
After multiplying this equation by $u$ and integrating over $D$, we obtain that $R(u)=0$; i.e., $u \in M$.

By assumptions (v) and (vi), $R$ is $C^{2}$-differentiable. To show that $M$ is a $C^{2}$ hypersurface, we need to show that $R^{\prime}(u) \neq 0$ for all $u \in M$.

Suppose there exists a $u \in M$ such that $R^{\prime}(u)=0$. Then $2(-\Delta u-f(u))=$ $u f^{\prime}(u)-f(u)$. After multiplying this by $u$ and integrating over $D$, we obtain that

$$
0=2 R(u)=\int_{D} u\left(u f^{\prime}(u)-f(u)\right) d x
$$

Therefore, $u=0$, which is impossible since $u \in M$. Hence, $M$ is a $C^{2}$-submanifold of $H_{0}^{1}(D)$ of codimension 1 .

(iv): Let $u \in M$ and $\alpha \in \mathbf{R}_{+}$be arbitrary. Then,

$$
\begin{aligned}
j(\alpha) & =\frac{1}{2} \alpha^{2} \int_{D}|\nabla u|^{2} d x-\int_{D} F(\alpha u) d x, \\
j^{\prime}(\alpha) & =\alpha \int_{D}|\nabla u|^{2} d x-\int_{D} u f(\alpha u) d x \\
j^{\prime \prime}(\alpha) & =\int_{D}|\nabla u|^{2} d x-\int_{D} u^{2} f^{\prime}(\alpha u) d x=\int_{D} u\left(f(u)-u f^{\prime}(\alpha u)\right) d x .
\end{aligned}
$$

Since $f$ is strictly convex, it follows that $j^{\prime}(\alpha)=0$ if and only if $\alpha=1$. Moreover, $j^{\prime \prime}(1)<0$.

(v): Let $u \in \tilde{M}$ and $\alpha \in \mathbf{R}_{+}$be arbitrary. Then,

$$
\begin{aligned}
r(\alpha) & =\alpha^{2} \int_{D}|\nabla u|^{2} d x-\int_{D} \alpha u f(\alpha u) d x \\
r^{\prime}(\alpha) & =2 \alpha \int_{D}|\nabla u|^{2} d x-\int_{D} u f(\alpha u) d x-\int_{D} \alpha u^{2} f^{\prime}(\alpha u) d x .
\end{aligned}
$$

Thus, since $R(u) \leq 0$ we obtain that

$$
\begin{aligned}
r^{\prime}(1) & =2 \int_{D}|\nabla u|^{2} d x-\int_{D} u f(u) d x-\int_{D} u^{2} f^{\prime}(u) d x \\
& =2 R(u)+\int_{D} u f(u) d x-\int_{D} u^{2} f^{\prime}(u) d x<0 .
\end{aligned}
$$

Therefore, for $\alpha$ close enough to $1, r(\alpha)$ is strictly monotone decreasing

(vi): Let $u \in H_{0}^{1}(D)-\{0\}$ and $\alpha \in \mathbf{R}_{+}$be arbitrary. Then

$$
\begin{aligned}
R(\alpha u) & =\alpha^{2} \int_{D}|\nabla u|^{2} d x-\int_{D} \alpha u f(\alpha u) d x \\
& \geq \alpha^{2}\|u\|^{2}-\varepsilon \alpha^{2}\|u\|_{2}^{2}-B \alpha^{q}\|u\|_{q}^{q} \\
& \geq \alpha^{2}\|u\|^{2}-\varepsilon \alpha^{2} K_{2}\|u\|^{2}-B \alpha^{q} K_{1}\|u\|^{q} \\
& =\alpha^{2} B K_{1}\|u\|^{q}\left(\frac{1-\varepsilon K_{2}}{B K_{1}}\|u\|^{2-q}-\alpha^{q-2}\right)
\end{aligned}
$$

for some $K_{1}, K_{2}>0$. So, $R(\alpha u)>0$ for $\alpha$ close to 0 , and $R(\alpha u)<0$ for $\alpha$ big enough. Hence, there exists an $\alpha_{0} \in \mathbf{R}_{+}$such that $R\left(\alpha_{0} u\right)=0$. Obviously, $\alpha_{0} \in[0,1]$ if $u \in \tilde{M}$. 
Uniqueness of $\alpha$ follows from the fact that for any $u \in H_{0}^{1}(D)-\{0\}$ the function $s$ from $\mathbf{R}_{+}$into $\mathbf{R}$ defined by

$$
s(\alpha)=\frac{1}{\alpha} \int_{D} u f(\alpha u) d x
$$

is strictly monotone.

All this implies that the function $p$ is well defined. Moreover, it is an even function, since $R$ is even. It remains to show continuity.

Let $u \in H_{0}^{1}(D)-\{0\}$. Let $p(u)=\alpha$. Since $R(\alpha u)=0$, we obtain that

$$
\alpha \int_{D}|\nabla u|^{2} d x=\int_{D} u f(\alpha u) d x
$$

and

$$
r^{\prime}(\alpha)=\int_{D} u f(\alpha u) d x-\int_{D} \alpha u^{2} f^{\prime}(\alpha u) d x \neq 0 .
$$

The statement then follows from the Implicit Function Theorem.

(vii): Let $u \in \tilde{M}$ and $\alpha \in \mathbf{R}_{+}$be arbitrary. Then,

$$
g(\alpha)=\int_{D}\left(\frac{1}{2} \alpha u f(\alpha u)-F(\alpha u)\right) d x
$$

and

$$
\begin{aligned}
g^{\prime}(\alpha) & =\frac{1}{2} \int_{D} u f(\alpha u) d x+\frac{1}{2} \int_{D} \alpha u^{2} f^{\prime}(\alpha u) d x-\int_{D} u f(\alpha u) d x \\
& =\frac{1}{2} \int_{D} u\left(\alpha u f^{\prime}(\alpha u)-f(\alpha u)\right) d x>0
\end{aligned}
$$

(viii): The statement follows directly from assumptions (vi), (ii), and part (i).

This completes the proof of the proposition.

It is known that the tangent space of $M$ at $u \in M$ can be identified with the following subspace of $H_{0}^{1}(D)$ :

$$
T_{u} M=\left\{v \in H_{0}^{1}(D) \mid d_{u} R(v)=\int_{D} R^{\prime}(u) v d x=0\right\},
$$

which also has codimension one.

Let $u \in M$ be arbitrary but fixed. Then for any $w \in H_{0}^{1}(D)$ there is a unique decomposition $w=\alpha R^{\prime}(u)+w^{T}$, where $w^{T}$ is the projection of $w$ into $T_{u} M$, and $\alpha=\left(w, R^{\prime}(u)\right) /\left\|R^{\prime}(u)\right\|^{2}$.

Obviously, we can obtain a Finsler structure on $M$ by assigning continuously to each point $u \in M$ the restriction of the inner product on $H_{0}^{1}(D)$ to $T_{u} M$.

2. Existence of infinitely many steady states. First we observe that the steady states of (1) are exactly the critical points of $J$. Since all of them are in $M$, it seems natural to apply the Ljusternik-Schnirelman category theory to $J_{\mid M}$ and to obtain infinitely many steady states (see $[3,4])$. In order to use the critical point theory we need the notion of genus.

DEFINITION 2.1. Let $\Sigma(M)$ be the set of all compact subsets of $M$, symmetric with respect to the origin. A set $A \in \Sigma(M)$ has genus $k$ (denoted by $\gamma(A)=k$ ), if $k$ is the smallest integer for which there exists an odd continuous map from $A$ into $\mathbf{R}^{k}-\{0\}$. We set $\gamma(A)=\infty$, if there is no such finite $k$, and $\gamma(\varnothing)=0$.

In the following proposition we state some properties of the genus. For the proofs see $[3,7]$. 
Proposition 2.2. Let $A, B \in \Sigma(M)$.

(1) If there exists an odd continuous map from $A$ into $B$, then $\gamma(A) \leq \gamma(B)$.

(2) If $A \subset B$, then $\gamma(A) \leq \gamma(B)$.

(3) If there exists an odd homeomorphism from $A$ into $B$, then $\gamma(A)=\gamma(B)$.

(4) $\gamma(A \cup B) \leq \gamma(A)+\gamma(B)$.

(5) If $\gamma(B)<\infty$, then $\gamma(\overline{A-B}) \geq \gamma(A)-\gamma(B)$.

(6) If $A$ is compact, then $\gamma(A)<\infty$, and there exists a $\delta$-neighborhood $N$ of $A$ such that $\gamma(N(A))=\gamma(A)$.

(7) Let $V$ be a k-dimensional subspace of $H_{0}^{1}(D)$ and $V^{\perp}$ its orthogonal complement. If $\gamma(A)>k$, then $A \cap V^{\perp} \neq \varnothing$.

The proof of existence of infinitely many critical points of $J$ in $M$ is based on an application of the Deformation Lemma. We will state it in a form which is useful for our purposes. The proof is a modification of the proofs given in $[\mathbf{4}, \mathbf{6}, \mathbf{1 1}]$.

DEFINITION 2.3. We say that $J_{\mid M}$ satisfies the Palais-Smale condition if any sequences $\left\{u_{n}\right\}$ in $M$ such that $\left\{J\left(u_{n}\right)\right\}$ is bounded, and $\left\|J_{\mid M}^{\prime}\left(u_{n}\right)\right\| \rightarrow 0$, contains a subsequence which is convergent in $M$.

Let $A_{b}=\{u \in M \mid J(u) \leq b\}$ and $K_{b}=\left\{u \in M \mid J(u)=b\right.$ and $\left.J_{\mid M}^{\prime}(u)=0\right\}$ for all $b \in \mathbf{R}$.

Observe that if $J_{\mid M}$ satisfies the Palais-Smale condition, then $K_{b}$ is compact.

LEMMA 2.4 (DEFORMATION LEMMA). Suppose $J_{\mid M}$ satisfies the PalaisSmale condition. Let $b \in \mathbf{R}$ and $U$ be any neighborhood of $K_{b}$. Then there exist constants $0<\varepsilon<\bar{\varepsilon}$ and a map $\eta \in C(M, M)$ such that

(i) $\eta(u)=u$ for all $u \in M$ with $|J(u)-b| \geq \bar{\varepsilon}$;

(ii) $\eta$ is an odd homeomorphism on $M$;

(iii) $J(\eta(u)) \leq J(u)$ for all $u \in M$;

(iv) $\eta\left(A_{b+\varepsilon}-U\right) \subset A_{b-\varepsilon}$;

(v) if $K_{b}=\varnothing$, then $\eta\left(A_{b+\varepsilon}\right) \subset A_{b-\varepsilon}$.

The following theorem is standard, and shows existence of infinitely many steady states. Its proof can be found in $[\mathbf{3}]$.

THEOREM 2.5. Let

$$
\Gamma_{k}=\{A \in \Sigma(M) \mid \gamma(A) \geq k\} \quad \text { and } \quad b_{k}=\inf _{A \in \Gamma_{k}} \sup _{u \in A} J(u) .
$$

Assume that $J_{\mid M}$ satisfies the Palais-Smale condition. Then $b_{k}$ is a critical value of $J_{\mid M}$ for all $k \geq 1$, and $0<\alpha \leq b_{1} \leq \cdots \leq b_{k} \leq b_{k+1} \cdots$ for some $\alpha>0$. If $b_{m}=b_{m+1}=\cdots=b_{m+r-1}=b$ for some $m, r \in \mathbf{N}$, then $\gamma\left(K_{b}\right) \geq r$.

In the following let $E_{0} \subset E_{1} \subset \cdots \subset E_{s} \subset \cdots$ be a sequence of finite-dimensional subspaces in $H_{0}^{1}(D)$ such that $\operatorname{dim} E_{s}=s$ and $\operatorname{cl}\left(\bigcup_{s=0}^{\infty} E_{s}\right)=H_{0}^{1}(D)$, where we denote by $\mathrm{cl}$ the closure in $H_{0}^{1}(D)$.

The following theorem shows the behavior of the critical values of $J_{\mid M}$. It also proves the existence of infinitely many critical points of $J$ in $M$.

THEOREM 2.6. $b_{k} \rightarrow \infty$ as $k \rightarrow \infty$.

Proof. Suppose $\left\{b_{k}\right\}$ is a bounded sequence in $\mathbf{R}$. Then there exists a constani $C>0$ such that for any $k \in \mathbf{N}$ we can find a set $A_{k} \in \Gamma_{k}$ which satisfies the 
inequalities

$$
0<\sup _{u \in A_{k} \cap E_{k-1}^{\perp}} J(u) \leq \sup _{u \in A_{k}} J(u) \leq b_{k}+\frac{1}{k}<C .
$$

Since $A_{k} \cap E_{k-1}^{\perp}$ is compact, there exists a point $u_{k} \in A_{k} \cap E_{k-1}^{\perp}$ such that

$$
J\left(u_{k}\right)=\sup _{u \in A_{k} \cap E_{k-1}^{\perp}} J(u)<C .
$$

In this way we obtain a sequence $\left\{u_{k}\right\}$ in $H_{0}^{1}(D)$ which is bounded by Proposition 1.1(ii). Therefore it contains a subsequence (denoted in the same way) which converges weakly in $H_{0}^{1}(D)$. Let $u$ be its limit.

Lower semicontinuity of weak limits implies that $R(u) \leq \liminf \inf _{k \rightarrow \infty} R\left(u_{k}\right)=0$.

Hence, $u \in \tilde{M}$, and therefore, by Proposition 1.1.(i), $u \neq 0$.

On the other hand, since $E_{1}^{\perp} \supset E_{2}^{1} \supset \cdots \supset E_{k}^{\perp} \supset \cdots \supset\{0\}$, we obtain that $u_{k} \rightarrow 0$. Hence, $u=0$, which is a contradiction. This proves the theorem.

Thus, in order to obtain infinitely many steady states, it is sufficient to show that $J_{\mid M}$ satisfies the Palais-Smale condition.

THEOREM 2.7. Let $\left\{u_{i}\right\}$ be a sequence in $M$ such that $\left\{J\left(u_{i}\right)\right\}$ is bounded and $\left\|J_{\mid M}^{\prime}\left(u_{i}\right)\right\| \rightarrow 0$ as $i \rightarrow \infty$. Then $\left\{u_{i}\right\}$ contains a subsequence which converges to a critical point of $J$.

Proof. Let $\left\{u_{i}\right\}$ be a sequence in $M$ such that $\left\{J\left(u_{i}\right)\right\}$ is bounded and $\left\|J_{\mid M}^{\prime}\left(u_{i}\right)\right\| \rightarrow 0$ as $i \rightarrow \infty$. Proposition 1.1(ii) implies that $\left\{u_{i}\right\}$ is a bounded sequence in $H_{0}^{1}(D)$, and therefore it contains a weakly convergent subsequence (that we denote in the same way). Let $u$ be its limit.

Since the imbedding $H_{0}^{1}(D) \hookrightarrow L^{q}(D)$ is compact, it follows that $u_{i} \rightarrow u$ in $L^{q}(D)$, and therefore

$$
\lim _{i \rightarrow \infty} \int_{D} u_{i} f\left(u_{i}\right) d x=\int_{D} u f(u) d x
$$

and

$$
\lim _{i \rightarrow \infty} \int_{D} u^{2} f^{\prime}\left(u_{i}\right) d x=\int_{D} u^{2} f^{\prime}(u) d x .
$$

Since $J_{\mid M}^{\prime}\left(u_{i}\right)=J^{\prime}\left(u_{i}\right)-\alpha_{i} R^{\prime}\left(u_{i}\right)$ for some $\alpha_{i} \in \mathbf{R}$, we obtain that

$$
\left(2 \alpha_{i}-1\right) \int_{D}\left(-\Delta u_{i}-f\left(u_{i}\right)\right) w d x-\alpha_{i} \int_{D}\left(u_{i} f^{\prime}\left(u_{i}\right)-f\left(u_{i}\right)\right) w d x \rightarrow 0
$$

as $i \rightarrow \infty$ for $w \in H_{0}^{1}(D)$.

In particular, it follows for $w=u_{i}$ that

$$
\left(2 \alpha_{i}-1\right) R\left(u_{i}\right)-\alpha_{i} \int_{D} u_{i}\left(u_{i} f^{\prime}\left(u_{i}\right)-f\left(u_{i}\right)\right) d x \rightarrow 0
$$

as $i \rightarrow \infty$. Therefore, since $R\left(u_{i}\right)=0$, we obtain that $\alpha_{i} \rightarrow 0$ as $i \rightarrow \infty$. Hence, $-\Delta u_{i}-f\left(u_{i}\right) \rightarrow 0$ as $i \rightarrow 0$ in $H^{-1}(D)$. Therefore,

$$
\int_{D}|\nabla u|^{2} d x=-\lim _{i \rightarrow \infty} \int_{D} u \Delta u_{i} d x=\lim _{i \rightarrow \infty} \int_{D} u f\left(u_{i}\right) d x=\int_{D} u f(u) d x .
$$

Thus, $R(u)=0$; i.e., $u \in M$. 
On the other hand, we obtain that

$$
\lim _{i \rightarrow \infty} \int_{D}\left|\nabla u_{i}\right|^{2} d x=\lim _{i \rightarrow \infty} \int_{D} u_{i} f\left(u_{i}\right) d x=\int_{D} u f(u) d x=\int_{D}|\nabla u|^{2} d x .
$$

This implies that $u_{i} \rightarrow u$ in $H_{0}^{1}(D)$.

In particular, $u$ is a critical point of $J$.

3. Instability of the steady states. Before discussing instability let us prove some preliminary propositions.

Proposition 3.1. Let $\Sigma(\tilde{M})$ be the set of all compact subsets of $\tilde{M}$, symmetric with respect to the origin. Let $\tilde{\Gamma}_{k}=\{A \in \Sigma(\tilde{M}) \mid \gamma(A) \geq k\}$. Let

$$
c_{k}=\inf _{A \in \tilde{\Gamma}_{k}} \sup _{u \in A}\left(J(u)-\frac{1}{2} R(u)\right) \text {. }
$$

Then $b_{k}=c_{k}$.

PROOF. Since $\Gamma_{k} \subset \tilde{\Gamma}_{k}$, we obtain that $c_{k} \leq b_{k}$. So, it remains to show that $b_{k} \leq c_{k}$.

By definition of $c_{k}$, for any $\varepsilon>0$ there is a set $A \in \tilde{\Gamma}_{k}$ such that

$$
c_{k} \leq \sup _{u \in A}\left(J(u)-\frac{1}{2} R(u)\right) \leq c_{k}+\varepsilon .
$$

Let $B=\{p(u) u \mid u \in A\}$, where $p$ is the map defined in Proposition 1.1(vi). Then $B \in \Gamma_{k}$, and by Proposition 1.1(vi),(vii),

$$
\begin{aligned}
b_{k} & \leq \sup _{u \in B} J(u)=\sup _{u \in A}\left(J(p(u) u)-\frac{1}{2} R(p(u) u)\right) \\
& \leq \sup _{u \in A}\left(J(u)-\frac{1}{2} R(u)\right) \leq c_{k}+\varepsilon .
\end{aligned}
$$

Since $\varepsilon$ was arbitrary, we obtain that $b_{k} \leq c_{k}$.

Proposition 3.1. Let $\phi$ be a critical point of $\left(J-\frac{1}{2} R\right)_{\mid \tilde{M}}$. Then $\phi \in M$ and $\phi$ is a critical point of $J$.

Proof. Suppose $\phi \in \tilde{M}-M$. Then

$$
J^{\prime}(\phi)-\frac{1}{2} R^{\prime}(\phi)=\frac{1}{2}\left(\phi f^{\prime}(\phi)-f(\phi)\right)=0 .
$$

Since $f$ is convex, it follows that $\phi=0$. This contradicts Proposition 1.1(i). Hence, $\phi \in M$.

Since $\phi$ is a critical point of $\left(J-\frac{1}{2} R\right)_{\mid \tilde{M}}$, there exists a Lagrange multiplier $\lambda \in \mathbf{R}$ such that $J^{\prime}(\phi)-\left(\frac{1}{2}+\lambda\right) R^{\prime}(\phi)=0$. Therefore,

$$
2 \lambda(-\Delta \phi-f(\phi))=\left(\frac{1}{2}+\lambda\right)\left(\phi f^{\prime}(\phi)-f(\phi)\right) .
$$

Multiplying the last equation by $\phi$ and integrating over $D$, we obtain that

$$
0=2 \lambda R(\phi)=\left(\frac{1}{2}+\lambda\right) \int_{D} \phi\left(\phi f^{\prime}(\phi)-f(\phi)\right) d x .
$$

Since $f$ is convex, it follows that $\lambda=-\frac{1}{2}$, and therefore, $J^{\prime}(\phi)=0$. 
Proposition 3.3. The set

$$
\Delta=\left\{B \in \Sigma(\tilde{M}) \mid \gamma(A) \geq k, J(u)<b_{k} \text { for all } u \in B\right\}
$$

is not empty.

PROOF. By definition of $b_{k}$, for any $\varepsilon>0$ there exists a set $A \in \Gamma_{k}$ such that $b_{k} \leq \sup _{u \in A} J(u)<b_{k}+\varepsilon$. From Proposition 1.1(viii) we obtain that

$$
\int_{D} u\left(u f^{\prime}(u)-f(u)\right) d x>S
$$

for all $u \in A$ and some $S>0$.

Let $\alpha>1$ but close to 1 . Let $u \in A$ be arbitrary. Consider the function $j(\alpha)=J(\alpha u)$. Since $j^{\prime}(1)=R(u)=0$ and

$$
j^{\prime \prime}(1)=\int_{D} u\left(f(u)-u f^{\prime}(u)\right) d x<-S,
$$

we obtain that

$$
J(\alpha u)<J(u)-\frac{1}{2} S(\alpha-1)^{2}<b_{k}+\varepsilon-\frac{1}{2} S(\alpha-1)^{2} .
$$

For $\varepsilon$ small enough there exists an $\alpha>1$ but close to 1 such that

$$
\varepsilon-\frac{1}{2} S(\alpha-1)^{2} \leq 0
$$

and therefore $J(\alpha u)<b_{k}$.

Consider the function $r(\alpha)=R(\alpha u)$, where $\alpha$ satisfies (*). By Proposition 1.1(v), $r$ is strictly monotone decreasing in a small neighborhood of 1 , and therefore for $\varepsilon$ small enough we obtain that $R(\alpha u)<0$.

Let $B=\{\alpha u \mid u \in A\}$, where $\alpha>1$ is such that $J(\alpha u)<b_{k}, R(\alpha u)<0$ for all $u \in A$. Then $B \in \Delta$. This completes the proof of the proposition.

In the following we denote by $u\left(t, u^{*}\right)$ the solution of (1) with the following properties:

(i) $u\left(0, u^{*}\right)=u^{*}$;

(ii) $R\left(u\left(0, u^{*}\right)\right)<0$;

(iii) $E\left(u\left(0, u^{*}\right), u_{t}\left(0, u^{*}\right)\right)<b_{k}$, where $E$ is the functional, describing energy, defined in the following way:

$$
E(u, v)=\frac{1}{2} \int_{D}|v|^{2} d x+\frac{1}{2} \int_{D}|\nabla u|^{2} d x-\int_{D} F(u) d x
$$

for all $(u, v) \in H_{0}^{1}(D) \times L^{2}(D)$.

Moreover, we denote by $T\left(u^{*}\right)$ the time of existence of $u\left(t, u^{*}\right)$.

The following statement gives a condition for a solution of (1) to blow up in finite time; it was proved by L. E. Payne and D. H. Sattinger in [13].

Proposition 3.4. Let $u^{*} \in M$ be such that $R\left(u\left(t, u^{*}\right)\right)<0$ for all $0 \leq t<$ $T\left(u^{*}\right)$. Then $u\left(t, u^{*}\right)$ blows up in finite time.

Thus, in order to prove instability of a steady state, it is sufficient to show that it can be approximated by a sequence $\left\{u_{i}^{*}\right\}$ such that $R\left(u\left(t, u_{i}^{*}\right)\right)<0$ for all $0 \leq t<T\left(u_{i}^{*}\right)$ and all $i$. This is the idea of the following theorem. 
THEOREM 3.5. There exists an unstable critical point of (1) which corresponds to the critical value $b_{k}, k \geq 2$. In any neighborhood of this critical point there exists a solution of (1) which blows up in finite time.

Let $\left\{A_{i}\right\}$ be a sequence in $\tilde{\Gamma}_{k}$ such that $J(u)<b_{k}$ and $-2 / i<R(u)<0$ for all $u \in A_{i}$. (Such sets can be obtained by the method used in Proposition 3.3.) Then

$$
b_{k} \leq \sup _{w \in A_{i}}\left(J(w)-\frac{1}{2} R(w)\right)<b_{k}+\frac{1}{i} .
$$

Let us denote by $u\left(t, u^{*}\right)$ the solution of (1) such that $u\left(0, u^{*}\right)=u^{*}$, and $u_{t}\left(0, u^{*}\right)=0$. Let

$$
\delta_{i}=b_{k}-\sup _{w \in A_{i}} J(w) .
$$

Then $R(w)>-3 \delta_{i}$ for all $w \in A_{i}$. Furthermore, let

and

$$
A_{i}^{0}=\left\{w \in A_{i} \mid R(u(t, w))>-\delta_{i} \text { for some } t \geq 0\right\}
$$

$$
t(w)= \begin{cases}\inf \left\{t \geq 0 \mid-\delta_{i}<R(u(t, w)) \leq 0\right\}, & \text { for } w \in A_{i}^{0}, \\ \inf \left\{t \geq 0 \mid R(u(t, w))<-3 \delta_{i}\right\}, & \text { for } w \in A_{i}-A_{i}^{0} .\end{cases}
$$

Furthermore, let

$$
\tilde{d}(t, w)= \begin{cases}u(t, w), & \text { for } t<t(w) \\ u(t(w), w), & \text { for } t \geq t(w)\end{cases}
$$

Since $A_{i}$ is compact, it follows that

$$
t^{*}=\sup \left\{t(w) \mid w \in A_{i}^{0}\right\}<\infty .
$$

Let $d(w)=\tilde{d}\left(t^{*}, w\right)$. Then $d$ is an odd continuous map from $A_{i}$ into $H_{0}^{1}(D)$ for all $i$. Therefore, $B_{i}=d\left(A_{i}\right) \in \tilde{\Gamma}_{k}$.

Since energy is conserved under the flow of (1), it follows that $J(w) \leq b_{k}-\delta_{i}$ for all $w \in B_{i}$. Therefore,

$$
J(w)-\frac{1}{2} R(w)<b_{k}-\delta_{i}+\frac{1}{2} \delta_{i}<b_{k}
$$

for all $w \in d\left(A_{i}^{0}\right) \subset B_{i}$.

Let $\tilde{B}_{i}=\left\{v \in B_{i} \mid J(v)-\frac{1}{2} R(v) \geq b_{k}\right\}$. Proposition 3.1 implies that $\tilde{B}_{i} \neq \varnothing$. Moreover, from the above argument we obtain that $\tilde{B}_{i} \subset d\left(A_{i}-A_{i}^{0}\right)$; i.e., $u(t, v)$ blows up in finite time for all $v \in \tilde{B}_{i}$.

Now we claim that for any neighborhood $U$ of $K_{b_{k}}$ there exists an integer $i$ such that $U \cap \tilde{B}_{i} \neq \varnothing$.

Let $U$ be any neighborhood of $K_{b_{k}}$. Since $f$ is strictly convex and $\tilde{M}$ is bounded away from zero, it follows that $\left(J-\frac{1}{2} R\right)_{\mid \tilde{M}}$ satisfies the Palais-Smale condition, and we can apply the Deformation Lemma (Lemma 2.4). Thus, there exists a homeomorphism $\eta \in C(\tilde{M}, \tilde{M})$ such that $\eta\left(A_{b_{k}+\varepsilon}-U\right) \subset A_{b_{k}-\varepsilon}$ for some $\varepsilon$ small enough.

Since $J(v)-\frac{1}{2} R(v)<b_{k}+2 \delta_{i}$ for all $v \in B_{i}$, choosing $i$ big enough we obtain that $\eta\left(B_{i}-U\right) \subset A_{b_{k}-\varepsilon}$. But, since $\eta\left(B_{i}\right) \subset \tilde{\Gamma}_{k}$, it follows that there exists a point $v_{i} \in U \cap B_{i}$ such that

Hence, $v_{i} \in U \cap \tilde{B}_{i}$.

$$
b_{k} \leq J\left(\eta\left(v_{i}\right)\right)-\frac{1}{2} R\left(\eta\left(v_{i}\right)\right) \leq J\left(v_{i}\right)-\frac{1}{2} R\left(v_{i}\right) .
$$


In this way we obtain a sequence $\left\{v_{i}\right\}$ in $H_{0}^{1}(D)$ such that $v\left(t, v_{i}\right)$ blows up in finite time, $J\left(v_{i}\right)-\frac{1}{2} R\left(v_{i}\right) \rightarrow b_{k}$ as $i \rightarrow \infty$, the sequence $\left\{v_{i}\right\}$ converges to a critical point $\phi$ of $\left(J-\frac{1}{2} R\right)_{\mid \tilde{M}}$; i.e., $\phi$ is a critical point of $J$ (see Proposition 3.2). This completes the proof of the theorem.

\section{REFERENCES}

1. H. Amann, Nonlinear operators in ordered Banach spaces and some applications to nonlinear boundary value problems, Nonlinear Operators and the Calculus of Variations (Bruxelles, 1975), Lecture Notes in Math., vol. 543, Springer, Berlin and New York, 1976.

2. A. Ambrosetti, On the existence of multiple solutions for a class of nonlinear boundary value problems, Rend. Sem. Univ. Padova 49 (1973), 195-204.

3. A. Ambrosetti and P. H. Rabinowitz, Dual variational methods in critical point theory and applications, J. Funct. Anal. 14 (1973), 349-381.

4. H. Berestycki and P. L. Lions, Nonlinear scalar field equations, Arch. Rational Mech. Anal. 82 (1983), 313-375.

5. F. E. Browder, Existence theorems for nonlinear partial differential equations, Proc. Sympos. Pure Math., vol. 16, Amer. Math. Soc., Providence, R. I., 1970, pp. 1-60.

6. D. C. Clark, A variant of the Lusternik-Schnirelman theory, Indiana Univ. Math. J. 22 (1972), 65-74.

7. C. V. Coffman, A minimum-maximum principle for a class of nonlinear integral equa tions, J. Analyse Math. 22, (1969), 391-419.

8. J. A. Hempel, Multiple solutions for a class of nonlinear elliptic boundary value problems, Indiana Univ. Math. J. 20 (1971), 983-996.

9. L. A. Ljusternik and L. G. Schnirelman, Méthodes topologique dans les problèmes variationels, Actualités Sci. Indust., No. 188, Hermann, Paris, 1934.

10. R. Nussbaum, Positive solutions of nonlinear elliptic boundary value problems, J. Math. Anal. Appl. (2) 51 (1975), 461-482.

11. R. S. Palais, Critical point theory and minimax principle, Proc. Sympos. Pure Math., vol. 15, Amer. Math. Soc., Providence, R. I., 1970, pp. 185-212.

12. _ Ljusternik-Schnirelman theory on Banach manifolds, Topology 5 (1966), 115-132.

13. L. E. Payne and D. H. Sattinger, Saddle points and instability of nonlinear hyperbolic equations, Israel J. Math. 22 (1975), 273-303.

14. P. H. Rabinowitz, Variational methods for nonlinear elliptic eigenvalue problems, Indiana Univ. Math. J. 23 (1973/74), 729-754.

15. J. Shatah, Stable standing waves of nonlinear Klein-Gordon equations, Comm. Math. Phys. 91 (1983), 313-327.

16. W. Strauss, Nonlinear invariant wave equations, Invariant Wave Equations (Erice, 1977), Lecture Notes in Physics, No. 73, Springer-Verlag, Berlin and New York, 1978, pp. 197-249.

17. H. Berestycki and P. L. Lions, Théorie des points critique et instabilité des ondes stationnaires pour des equations de Schrödinger non-linéaires, C. R. Acad. Sci. Paris, 1985.

18. H. Berestycki and T. Cazenave, Instabilité des états stationnaire dans les équations de Schrödinger et de Klein-Gordon non-linéaires, C. R. Acad. Sci., Paris, 1981, pp. 489-492.

19. D. H. Sattinger, Topics in stability and bifurcation theory, Lecture Notes in Math., vol. 309, Springer, 1973.

20. J. Shatah, Unstable ground states of nonlinear Klein-Gordon equations, Trans. Amer. Math. Soc. 290 (1985).

Lefschetz Center for Dynamical Systems, Division of Applied MathematICS, BROWN UNIVERSITY, PROVIDENCE, RHODE ISLAND 02912 